\title{
Providing Psychiatric Care for an Expanding Population of Cancer Survivors: Imperatives for Psychiatric Education and Leadership
}

\author{
Mary K. Morreale ${ }^{1}$. Richard Balon ${ }^{1} \cdot$ Eugene V. Beresin $^{2} \cdot$ John H. Coverdale ${ }^{3}$. \\ Adam Brenner ${ }^{4}$. Anthony Guerrero ${ }^{5}$ - Alan K. Louie $^{6} \cdot$ Laura W. Roberts $^{6}$
}

Received: 17 November 2016 / Accepted: 22 November 2016/Published online: 5 December 2016

(C) Academic Psychiatry 2016

The number of cancer survivors, defined as people from the point of cancer diagnosis through end of life regardless of treatment outcome, is currently greater than 15 million and predicted to exceed 20 million by 2026 [1]. For patients in the midst of treatment for cancer, the prevalence of psychiatric diagnoses is approximately $50 \%$, with the majority being diagnosed with adjustment disorder [2]. For people on surveillance for the recurrence of cancer, myriad potential issues may cause psychological distress, including (but certainly not limited to) cognitive changes secondary to systemic chemotherapy, disfigurement, chronic pain, sexual dysfunction and infertility, and depression and anxiety related to fear of recurrence and ultimately death. Recognition is growing that effectively addressing the behavioral and psychosocial components of cancer care is key to improving overall outcomes. Caring for cancer survivors is an increasingly important responsibility for the profession of psychiatry, and preparing psychiatric residents and fellows to care for people living with and surviving cancer is an increasingly important responsibility in academic psychiatry.

Who is caring for, or will care for, this large number of patients? Although fellows trained in hematology, medical

Mary K. Morreale

mmorreale@wayne.edu

Wayne State University School of Medicine, Detroit, MI, USA

2 Harvard Medical School, Boston, MA, USA

Baylor College of Medicine, Houston, TX, USA

4 University of Texas Southwestern Medical Center, Dallas, TX, USA

5 University of Hawai'i John A. Burns School of Medicine, Honolulu, HI, USA

6 Stanford University, Stanford, CA, USA oncology, hospice, and palliative medicine are expected per Accreditation Council for Graduate Medical Education (ACGME) requirements to manage some behavioral components of care, interacting with psychologists and psychiatrists as they learn to do so is not obligatory $[3,4]$. Psychiatry is one of the specialties allowed to train in palliative medicine, but according to the American Board of Psychiatry and Neurology, only 110 psychiatrists have been certified between the years 2000 and 2015 [5]. The subspecialty of psycho-oncology, which began formally during the 1970s, has the stated goal of "incorporating the psychological, social, spiritual and existential dimensions and seeking to help the patient find a tolerable meaning to the presence of the unwelcome intruder of serious illness and threat to the future and to life itself," but it does not have associated formal fellowship training [6]. Psychosomatic medicine fellowships may lean heavily toward training in cancer care, for example, the combined program at the Memorial Sloan Kettering and New York Presbyterian Hospital, but ACGME program requirements in psychosomatic medicine do not require any specific exposure to oncologic populations $[7,8]$. In addition, the number of psychosomatic medicine fellows produced yearly (e.g., 87 in 2015 2016) is certainly not enough to manage the large number of cancer survivors [7].

Are general psychiatric residents currently well prepared to treat cancer survivors? The ACGME requires 2 months of consultation-liaison psychiatry, but, as in psychosomatic medicine, treatment of cancer patients is not guaranteed to be a core part of this experience [9]. In addition, if encounters with patients who have cancer do occur in these settings, psychiatric residents are more likely to focus on the acute psychiatric needs of this population than on longitudinal concerns. Most cancers occur in individuals over the age of 70 years, but only 1 month of focused experience is required in geriatric psychiatry $[9,10]$. It is entirely possible that a resident graduating from a program without a large associated cancer center or without considerable exposure 
to patients with cancer would not be adequately prepared to manage the psychiatric comorbidities of cancer.

So where does this leave the expanding population of cancer survivors? One of us (MKM) discussed this issue with Jimmie Holland, M.D., a noted researcher and chair of psychiatric oncology at the Memorial Sloan Kettering Cancer Center in New York, and several ideas emerged (personal communication, October 10, 2016). The first and foremost idea is to target both the shortage of psychiatrists in the USA and the low rates of mental health reimbursement, because these issues face patients seeking psychiatric care regardless of diagnosis [11]. According to Dr. Holland, one way to address these issues is by introducing a collaborative care model - an evidence-based, integrated approach for providing medical and behavioral health care within one system [12]. In such a model, primary care providers, care managers, and psychiatric consultants work together in an established method of treatment involving close monitoring of patients with validated clinical rating scales, "analogous to how patients with diabetes are monitored via HbAlc laboratory tests" [12]. Adjustments to treatment are made by the primary care team, which includes primary care providers (e.g., family practitioners, internists, nurse practitioners, and physician assistants) and care managers (e.g., nurses, social workers, and psychologists trained to coordinate care and provide brief behavioral interventions) [12]. Those patients who do not respond to treatment or who have an acute crisis can be referred to the psychiatric consultant, although at present, only a relatively small fraction of patients in collaborative care programs are referred [12]. Collaborative care models have been shown to be more effective than usual care for depression, anxiety, bipolar disorder, and schizophrenia and "can overcome the clinical inertia that is often responsible for ineffective treatment of common mental disorders in primary care" [12]. If a model like this were incorporated into the oncologist's office or cancer center, perhaps led by a psychiatrist trained in psychosomatic medicine, many more psychiatric comorbidities might be treated effectively for patients with cancer.

This model of optimal collaborative care is an ideal which educators alone may have difficulty advocating for. It is incumbent upon educators to work with chairs of departments and hospital administrators who may have the vision and resources to implement such valuable programs. What more can medical student and resident educators and residency and fellowship directors do? In didactic learning, it is feasible to add lectures related to psychiatric comorbidities associated with cancer care into medical student, resident, and fellowship curricula. Such educational efforts should be directed not only toward students training directly in psychiatry but also toward students training in hematology, oncology, hospice, and palliative medicine. Medical educators and training directors can encourage medical students who express interest in psychiatry to consider electives in oncology or psycho-oncology wherever possible and can encourage residents who are drawn to consultation-liaison psychiatry to pursue fellowships in psychosomatic medicine. In addition, medical educators and training directors can help residents interested in hospice and palliative medicine fellowships to structure their training to allow a smooth transition into this subspecialty. Expanding the requirements for consultation-liaison and geriatric exposure during general psychiatric residency might also be considered. Such a step would benefit not only patients with cancer but also the aging population as a whole, which can be expected to have multiple co-occurring medical and psychiatric conditions. Finally, the amount of time currently required in traditional inpatient and outpatient settings could be decreased, and a requirement might be considered for residents to practice in integrated settings with primary care physicians. When these opportunities do not exist, chairs might be persuaded to pursue collaborative care models if they are presented with data to support these methods of health care delivery [13].

\section{Compliance with Ethical Standards}

Disclosures On behalf of all authors, the corresponding author states that there is no conflict of interest.

\section{References}

1. Miller KD, Siegel RL, Lin CC, et al. Cancer treatment and survivor statistics. CA Cancer J Clin. 2016;66:271-81.

2. Holland JC, Alici Y. Management of distress in cancer patients. J Support Oncol. 2010;8:4-12.

3. Accreditation Council for Graduate Medical Education. ACGME program requirements for graduate medical education in hematology and medical oncology (internal medicine). Available at http://www. acgme.org/portals/0/pfassets/programrequirements/155 hematology_oncology_int_med_2016.pdf. Last accessed $\overline{1}$ November 2016.

4. Accreditation Council for Graduate Medical Education. ACGME program requirements for graduate medical education in hospice and palliative medicine (anesthesiology, family medicine, internal medicine, pediatrics, psychiatry, or radiation oncology). Available at http://www.acgme.org/Portals/0 /PFAssets/ProgramRequirements/540_hospice_and_palliative medicine_2016_1-YR.pdf. Last accessed 1 November 2016.

5. American Board of Psychiatry and Neurology. ABPN certifications by year-subspecialties. Available at https://www.abpn.com/wpcontent/uploads/2016/08/ABPN-Certifications-by-YearSubspecialties.pdf. Last accessed 1 November 2016.

6. Holland JC. History of psycho-oncology: overcoming attitudinal and conceptual barriers. Psychosom Med. 2002;64:206-21.

7. Accreditation Council for Graduate Medical Education. AGME programs by specialty. Available at https://apps.acgme. org/ads/Public/Programs/Search?stateId=\&specialtyId=139 \&city=. Last accessed 23 October 2016.

8. Accreditation Council for Graduate Medical Education. ACGME program requirements for graduate medical education in psychosomatic medicine. Available at https://www.acgme.org/Portals/0 /PFAssets/ProgramRequirements/409_psychosomatic med_2016_1-YR.pdf. Last accessed 23 October 2016. 
9. Accreditation Council for Graduate Medical Education. Program requirements for graduate medical education in psychiatry. Available at https://www.acgme.org/Portals/0 /PFAssets/ProgramRequirements/400_psychiatry_2016.pdf. Last accessed 23 October 2016.

10. American Cancer Society: cancer facts and figures. Available at http://www.cancer.org/acs/groups/content/@research/documents /document/acspc-047079.pdf. Last accessed 23 October 2016.

11. Konrad TR, Ellis AR, Thomas KC, et al. County-level estimates of need for mental health professionals in the United States. Psychiatr Serv. 2009;60:1307-14.
12. Unützer J, Harbin H, Michael Schoenbaum, Druss B. The collaborative care model: an approach for integrating physical and mental health care in Medicaid health homes. May 2013. Available at http://medicaid.gov/State-ResourceCenter/Medicaid-State-Technical-Assistance/Health-HomesTechnical-Assistance/Downloads/HH-IRC-Collaborative-5-13. pdf. Last accessed 22 October 2016.

13. Archer J, Bower P, Gilbody S, et al. Collaborative care for depression and anxiety problems. Cochrane Database Syst Rev. 2012. doi:10.1002/14651858.CD006525.pub2. 